\title{
Resource Allocation in a Cellular CDMA Environment
}

\author{
R. Bolla, F. Davoli, M. Perrando \\ Department of Communications, Computer and Systems Science (DIST), University of \\ Genoa, Via Opera Pia 13, I-16145 Genoa, Italy \\ \{lelus, franco, perr\}@dist.unige.it
}

Key words: Call admission control, CDMA, resource allocation, fixed point, cellular systems.

\begin{abstract}
A cellular environment is considered, based on CDMA. The main goal is to optimize the overall system capacity, in order to equalize and minimize the blocking probability of new calls entering into the system; at the same time, a constraint on the outage probability and, possibly, another one on handoff blocking are enforced. The control is based on the multiple access interference threshold, and the optimization algorithm tries to find the best choice of the admittance thresholds, based on the "a priori" knowledge of the traffic characteristics, and of a mobility model of the users.
\end{abstract}

\section{INTRODUCTION}

The growing user demand and the diversification of services in mobile wireless networks are stimulating the deployment of systems with smaller cell size and higher potential flexibility in the allocation of the resources, both across different services and among groups of cells. Such dynamic resource allocation aims at maintaining Quality of Service (QoS) requirements, even in the presence of traffic flows with different statistical nature and performance characteristics and of variable user mobility patterns. Notwithstanding the multiple access method adopted, some basic factors that play a key role in the QoS provisioning to multimedia traffic are the adaptation to user mobility, the provision of differentiated (pertraffic-class) resource allocation and the overall system's organization into coordinated management domains. In this respect, control actions can be applied at different operational layers in the network structure. 
In particular, bandwidth allocation can be considered at the channel level, in conjunction with the multiple access protocol, in different multiple access (TDMA or CDMA) environments (see, e.g., [1], [2], [10], [5]), or at a "higher" hierarchical layer, affecting the network structure and in conjunction with Call Admission Control (CAC); see [12], [9], [3], [4], among others. A control architecture based on a hierarchical paradigm, which reflects a possible network organization in cells and cell clusters, has been considered by the authors in a pure TDMA context [3], [4]. In this paper we consider a similar control structure, but embedded in a CDMA scheme, which is the preferred choice in the emerging UMTS [11].

We combine two mechanisms that operate at different levels within the network: i) a threshold-based admission control within the cell, which sets different acceptance levels for new calls originating in the cell and handoff calls coming from adjacent ones; ii) a dynamic resource sharing scheme at the cluster level, for the distribution of the system's capacity (in terms of the maximum number of calls compatible with a given multiple access interference) among the cells in a cluster.

The paper is organized as follows. We describe our model of the CDMA channel in the next section. In Section 3, we introduce the resource allocation mechanism and a numerical approximation to compute the Multiple Access Interference (MAI) with a given set of thresholds. In Section 4, we report and discuss several numerical results deriving from the application of the proposed scheme. Section 5 contains the conclusions.

\section{THE MODEL OF THE CELLULAR ENVIRONMENT}

In the following we give a specification of the environment considered in the present work, and also some hypotheses that have been taken into account in order to simplify calculations. A very similar model has been used in [6].

A cellular environment has been considered, in which Base Stations (BS's) perform a perfect power control on the Mobile Stations (MS's) under their domain; furthermore, exogenous (environmental, Gaussian, etc.) noise has been ignored, with respect to the endogenous interference. Voice activity detection has not been adopted in the present version of the work: a mobile transmits continuously for the whole duration of its call.

The total area is divided into $C$ hexagonal cells all of equal size, and the BS's antenna is omnidirectional and placed in the center of the cell. 
A MS is controlled by the BS which has the best radio propagation factor with that MS.

There is a complete separation between the forward and the reverse link, so that there is no interference between BS's and MS's.

The radio propagation model assumed is a generally accepted one [7], where the total attenuation depends on two variables: a random shadowing factor, log-normal distributed, and a path loss factor, depending on the $\alpha$ power of the distance. In other words, the attenuation factor suffered by a MS at distance $r$ from a BS is:

$$
\theta(r)=10^{\xi / 10} r^{-\alpha}
$$

where $\xi$ is a Gaussian random variable with standard deviation $\sigma$. The values for $\sigma$ and $\alpha$ generally adopted, and also considered in the present work are $\sigma=8$, and $\alpha=4$.

The new call (not originated by MS movements) arrival process is modeled, at a generic cell $i$, as an independent Poisson process with mean arrival rate $\lambda_{i}^{n c}$, whereas the mean call lifetime is exponentially distributed with mean duration of $1 / \mu$.

The model of MS mobility is analogous to [4]: a mobility coefficient $\gamma_{i}$, depending on the cell site, gives the fraction of calls that leave cell $i$ because they handed-off to another site, as explained more in detail in the following. At the boundary of the system a hand-off flow of entering calls is also considered.

The admission scheme is based on the measure of the Multiple Access Interference Ratio (MAI) estimated by every BS, supposed to be measured exactly. The admission control policy may operate differently, depending on the fact that the call requesting admission is generated by a hand-off situation, or it is a new call entering the system. Calls are admitted into the system if the instantaneous MAI is under a determined level. This MAI threshold may be different for the hand-off case and the new call case.

Given the parameters of the spread spectrum modulation, such as the processing gain, we can estimate the maximum value for the MAI above which the quality of the transmission falls under a minimum requisite. Let $M A I_{0}$ be this value. The probability that the cell MAI overcomes $M A I_{0}$ will be called outage probability.

\section{THE ALLOCATION PROCEDURE}

The main goal of this work is to maintain the outage probability in every single cell of the system under a given constraint $\hat{P}^{\text {otg }}$. In addition to this, another constraint $\hat{P}^{b, h o}$ may be imposed over the blocking probability of the calls entering for hand-off. This last constraint may 
be applied only in the case of different thresholds for hand-off calls and new calls.

Let us see in detail how the MAI is calculated over the system. If $k$ is the number of active calls in cell $i$, the total power $I(i)$ received by BS $i$ is given by:

$$
\begin{aligned}
I(i) & =\sum_{u \in\{\text { all MS's }\}} I_{u}(i)= \\
& =S k+S \sum_{j \neq i} \sum_{u \in\{\text { MS's not in cell } i\}}\left(\frac{r_{j}^{u}}{r_{i}^{u}}\right)^{\gamma} 10^{\left(\xi_{i}^{u}-\xi_{j}^{u}\right) / 10}= \\
& =I_{i n t}(k)+I_{e x t}(i)
\end{aligned}
$$

where $I_{u}(i)$ is the power of the signal received by BS $i$ from a MS $u$ transmitting to its own BS $j ; r_{j}^{u}$ and $\xi_{j}^{u}$ are the distance and the shadowing, respectively, of MS $u$ with respect to its own BS $j ; r_{i}^{u}$ and $\xi_{i}^{u}$ are the distance and the shadowing, respectively, of MS $u$ with respect to BS $i$; and $S$ is the power received by a BS from a perfectly power-controlled MS. Furthermore, $I_{\text {int }}(k)$ is defined as the contribution originated by $k$ MS's directly controlled by a BS, while $I_{\text {ext }}(i)$ is defined as the interference caused on BS $i$ by all the MS's not controlled by that BS.

The MAI measured at $\mathrm{BS} i$, having $k$ active calls, thus results:

$$
M A I(i, k)=\frac{I(i)-S}{S}=k-1+M A I_{e x t}(i)
$$

where we have defined

$$
M A I_{e x t}(i)=\frac{I_{e x t}(i)}{S}
$$

Even assuming a spatially uniform distribution of the users over the ground, each of the variables $M A I_{\text {ext }}(i)$, clearly depends on the global status of all the cells in the system; in other words, it depends on the number of calls being active in every cell, or, more precisely, on their joint distribution. In order to approximate the MAI of a given cell, with a quantity dependent only from the number of calls active in such cell, we remove the dependence on the status of other cells in the system, by considering each variable $M A I_{\text {ext }}(i)$ as a stochastic variable, having its own distribution. This distribution is then evaluated, assuming to know the steady state probability of the number of active calls in every cell.

In order to calculate the distribution of $M A I_{\text {ext }}(i)$, we consider the contribution to the MAI suffered by a generic $\mathrm{BS} i$, given by a single 

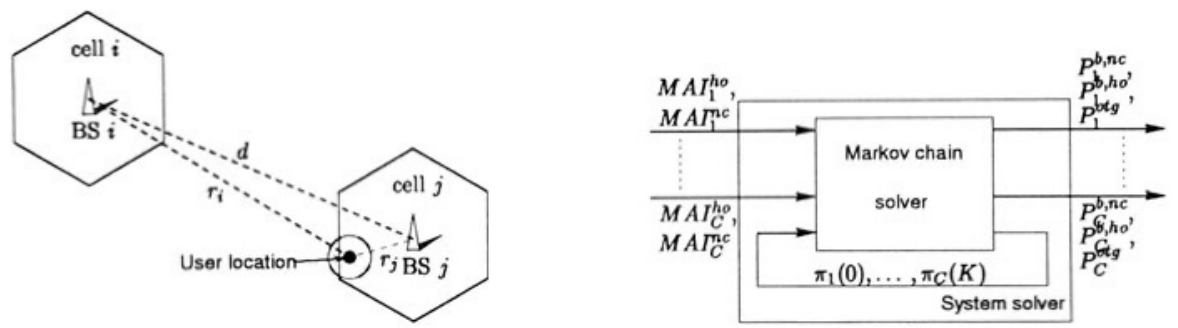

Figure 1 (a) MAI of a single user; (b) Fixed point solution

active call, which is running in a cell under the control of BS $j$. We call $M A I_{\text {single }}(d)$ this contribution, because it depends only on the relative distance $d$ between the two BS's, and its value is given by [7]:

$$
M A I_{\text {single }}(d)=\frac{1}{A} \int_{\text {cell } j} \mathcal{M}\left(r_{i}, r_{j}\right) d A
$$

where $A$ is the area of a cell, $r_{i}$ and $r_{j}$ are the distance of the area $d A$ from BS $i$ and BS $j$, respectively (see Figure 1 (a)), and

$$
\mathcal{M}\left(r_{i}, r_{j}\right)=\left(\frac{r_{j}}{r_{i}}\right)^{\alpha}\left\{10^{\left(\xi_{i}-\xi_{j}\right) / 10}\right\} \Phi\left(\xi_{i}-\xi_{j}, \frac{r_{j}}{r_{i}}\right)
$$

where

$$
\Phi\left(\xi_{i}-\xi_{j}, \frac{r_{j}}{r_{i}}\right)= \begin{cases}1, & \text { if }\left(r_{j} / r_{i}\right)^{\alpha} 10^{\left(\xi_{i}-\xi_{j}\right) / 10} \leq 1 \\ 0, & \text { otherwise }\end{cases}
$$

Now, as in [7], we consider the $M A I_{s}(d)$ as a Gaussian variable having its own mean and variance. Then, following an analogous procedure, we numerically calculate (from (5)) both the mean value and the variance.

As already stated, we suppose to know the steady state probability of the number of active calls in the system. $\pi_{j}(k)$ being the probability of having $k$ active calls in the generic cell $j$, we can write $M A I_{e x t}(i)$ as

$$
\begin{aligned}
M A I_{\text {ext }}(i) & =\sum_{j \neq i} \sum_{k=0}^{K} M A I_{\text {single }}\left(d_{i, j}\right) k \pi_{j}(k)= \\
& =\sum_{j \neq i} M A I_{\text {single }}\left(d_{i, j}\right) \bar{n}_{j}
\end{aligned}
$$

where $\bar{n}_{j}$ is the average number of active calls in cell $j, d_{i, j}$ is the distance between the two BS's, and $K$ is a "large enough" value for the maximum 
number of active calls that can be present in every cell, so that the probability of having more then $K$ active calls is zero. A good choice for $K$ may be the capacity of a single isolated cell: due to non null interference of other cells, the CAC cannot bring the number of active calls over this value.

Since $M A I_{\text {ext }}(i)$ is the sum of Gaussian distributed variables, it has a Gaussian distribution having as mean value the sum of mean values of the factors, and, as the variance, the sum of the variances of the factors. Let the outage probability suffered by cell $i$ be

$$
w_{i}^{\text {otg }}(k)=\operatorname{Pr}\left\{M A I(i, k)>M A I_{0}\right\}
$$

The control variables that decide the admission of calls are defined as:

$$
q_{i}^{n c}(k)= \begin{cases}1 & \text { if } M A I(i, k+1)<M A I_{i}^{n c} \\ 0 & \text { otherwise }\end{cases}
$$

representing the acceptance for a new call if $q_{i}^{n c}=1$ at cell $i$ and

$$
q_{i}^{h o}(k)= \begin{cases}1 & \text { if } M A I(i, k+1)<M A I_{i}^{h o} \\ 0 & \text { otherwise }\end{cases}
$$

representing the acceptance of a call entering cell $i$ for hand-off if $q_{i}^{h o}=1$.

Defining

$$
\begin{aligned}
& w_{i}^{h o}(k)=\operatorname{Pr}\left\{q_{i}^{h o}(k)=1\right\} \\
& w_{i}^{n c}(k)=\operatorname{Pr}\left\{q_{i}^{n c}(k)=1\right\}
\end{aligned}
$$

then, the total load entering the generic cell $i$ is

$$
\lambda_{i}(k)=\lambda_{i}^{h o} w_{i}^{h o}(k)+\lambda_{i}^{n c} w_{i}^{n c}(k)
$$

where the quantities $\lambda_{i}^{n c}$ represent the arrival rates of the calls originated by a new generation (not by hand-off), and $\lambda_{i}^{h o}$ represent the rates of calls that enter the cell for hand-off. These quantities are reduced, by the effect of the CAC policy, of a factor $w_{i}^{n c}(k)$ and $w_{i}^{h o}(k)$, respectively.

While the quantities $\lambda_{i}^{n c}, i=1, \ldots, C$ are given data of the problem, each $\lambda_{i}^{h o}$ depends on the state of the whole cellular environment, as well as on the mobility model.

Under the previous assumption we can calculate the acceptance factors in (10) and (11), and, consequently, the fraction of the accepted calls in (12). But now, in order to evaluate $\lambda_{i}(k)$ in (13), we need also the quantities $\lambda_{i}^{h o}$. The latter may be found from the mobility model of users, which will be now explained in detail. 

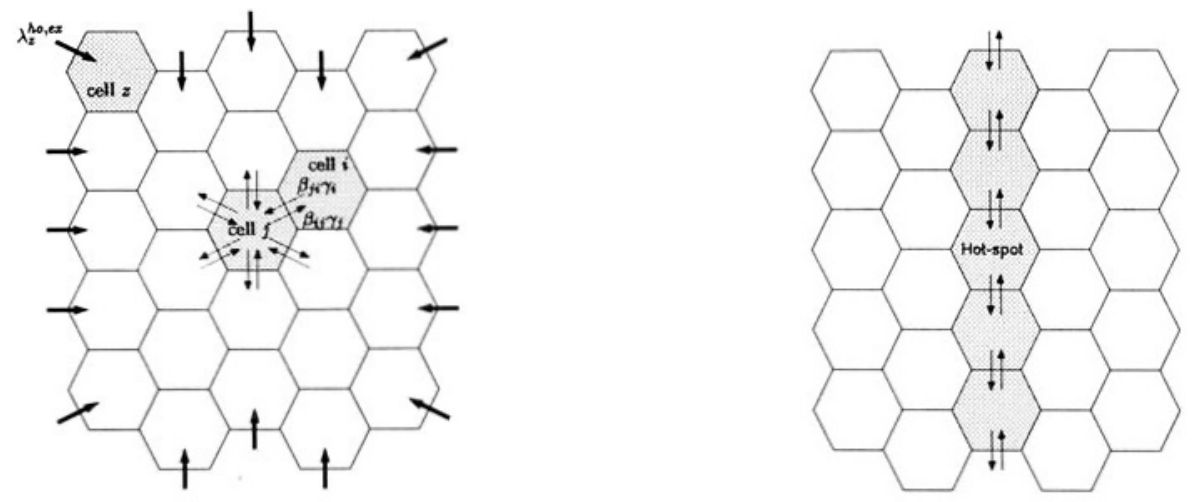

Figure 2 (a) Mobility model; (b) Optimization scenario

As already stated, every cell $i$ has a mobility factor $\gamma_{i}$, that indicates the rate of calls that die for hand-off. Being $\mu$ the mean call death rate for "natural termination", we can state that, given $k$ active calls within a cell, the call death rate is, for that cell, $k \mu$. The role of $\gamma_{i}$ is to state the call death rate for hand-off; more precisely, this rate is, for cell $i$, having $k$ active calls,

$$
\mu_{i}^{h o}(k)=\gamma_{i} \mu k
$$

By this, the total death rate for cell $i$ having $k$ active calls is

$$
\mu_{i}(k)=\left(1+\gamma_{i}\right) \mu k
$$

Furthermore, we need to know what is the part of the total exiting calls that goes to each of the neighboring cells. The part of traffic that exits from cell $j$ and goes to the adjacent cell $i$ is given by the coefficient $\beta_{i j}$, for which the following equations rnust be satisfied

$$
\begin{aligned}
\beta_{i j} & =0 \quad \text { if } i \notin \mathcal{A}(j) \\
\sum_{i \in \mathcal{A}(j)} \beta_{i j} & =1
\end{aligned}
$$

where $\mathcal{A}(j)$ is the set of cells adjacent to cell $j$.

An exogenous hand-off rate deriving from users that enter the cell cluster from outside is also considered for every border cell. Thus, for every cell $i$, an additional rate of $\lambda_{i}^{h o, e x}$ is taken into account, with the caution to set $\lambda_{i}^{h o, e x}=0$ if $i$ is not a border cell.

A scheme for mobility is illustrated in Figure 2 (a), where the behavior of one cell is detailed. 
The total rate of calls entering cell $i$ for hand-off is thus:

$$
\begin{aligned}
\lambda_{i}^{h o} & =\sum_{j \in \mathcal{A}(i)} \beta_{i j} \sum_{k=0}^{K} \mu_{j}^{h o}(k) k \pi_{j}(k)+\lambda_{i}^{h o, e x}= \\
& =\sum_{j \in \mathcal{A}(i)} \beta_{i j} \gamma_{j} \mu \bar{n}_{j}+\lambda_{i}^{h o, e x}
\end{aligned}
$$

where $\bar{n}_{j}=\sum_{k=0}^{K} k \pi_{j}(k)$ is the mean value of the number of active calls in cell $j$.

Now we have all the elements $\left(\lambda_{i}(k)\right.$ and $\left.\mu_{i}(k)\right)$ to solve the Markov chain for every cell $i$ and to find out the steady state probability $\pi_{i}(k)$ of having $k$ active calls in cell $i$.

In other words, we are trying to solve a vectorial fixed point equation in the form:

$$
x=f(x)
$$

where $x=\operatorname{col}\left[\pi_{1}(0), \ldots, \pi_{C}(K)\right]$.

Due to the very high complexity of function $f(\cdot)$, it is impossible to solve explicitly such an equation. As in [4], the assumption of existence of a single fixed point is made. This assumption is also supported from the work of $[8]$.

Starting from a random value for the $\pi_{i}(k)$, we may calculate all the values needed in order to recompute these probabilities. Repeating these steps until some norm of the $\pi_{i}(k)$ converges, gives rise to the solution of the equation. A graphical scheme illustrating these procedure can be found in Figure 1 (b). Now we can calculate the blocking probabilities of new calls and hand-off calls, and the outage probability of every cell:

$$
\begin{aligned}
P_{i}^{b, n c} & =\sum_{k}\left(1-w_{i}^{n c}(k)\right) \pi_{i}(k) \\
P_{i}^{b, h o} & =\sum_{k}\left(1-w_{i}^{h o}(k)\right) \pi_{i}(k) \\
P_{i}^{\text {otg }} & =\sum_{k} w_{i}^{\text {otg }}(k) \pi_{i}(k)
\end{aligned}
$$

The variables that remain fixed during this process are the admission thresholds $M A I_{i}^{n c}$ and $M A I_{i}^{h o}$. These are the control variables that we can use to optimize the capacity among different cells. The optimization goal is to minimize a global cost represented by the maximum blocking probability of the new calls

$$
P^{b, n c}=\max _{i}\left\{P_{i}^{b, n c}\right\}
$$


In other words, we want to solve the following problem

$$
\min _{M A I_{1}^{n c}, M A I_{1}^{h o}, \ldots, M A I_{C}^{n c}, M A I_{C}^{h o}}\left\{P^{b, n c}\right\}
$$

In this scenario we set a constraint over the quality, imposing a minimum level on the quality of the call at "transmission level", by requiring that the outage probability must remain under a certain threshold:

$$
P^{o t g}=\max _{i}\left\{P_{i}^{o t g}\right\} \leq \hat{P}^{o t g}
$$

An additional analogous constraint may be imposed over the blocking probabilities of hand-off calls, in order to ensure a "call-level" quality for calls that are running. In such a case, we set the following constraint

$$
P^{b, h o}=\max _{i}\left\{P_{i}^{b, h o}\right\} \leq \hat{P}^{b, h o}
$$

Otherwise, we may treat hand-off and new call connections equally. In this case, there is no difference between new or hand-off calls; thus, both are considered as new calls, and only the value of $P^{b, n c}$ is taken into account, paying attention to the fact that, in this case, it represents the blocking probability of both new and hand-off calls.

\section{OPTIMIZATION RESULTS}

We call case $\mathbf{A}$ the case for which no constraint on the hand-off blocking probability is set, and an identical CAC policy is adopted for both kinds of connections, and case $B$ the one for which we set a constraint on the maximum rate of blocked hand-off calls. In the latter the maximum blocking probability of $10^{-3}$ is imposed.

The optimization consists of solving a constrained mathematical programming problem with non-differentiable cost function, over a domain in a $2 C$-dimensional space, in case of different thresholds for hand-off and new calls, and over a $C$-dimensional space in the other case.

The procedure adopted consists of a kind of genetic algorithm, that generates a new population, taking into account a heuristic that tries to optimize the cost on every single cell.

The scenario we take into account consists of a $5 \times 5$ cell set, within which the possible hot-spot cell is the central one. Furthermore, the presence of a traffic lane is considered in the sense of the arrows. The latter is modeled with both an increment of users mobility and new call generation rate. All this can be depicted as in Figure 2 (b).

There are many parameters that can change the final result of the optimization, such as: mean load of the system, presence or absence of 
a hot-spot cell, mobility factor of the users, presence of a high hand-off rate in one predominant direction, etc.

Several cases have been taken into account, for which, unless otherwise indicated, the mobility coefficient is $\gamma_{i}=0.3 \forall i$, the fraction of users that exit towards every adjacent cell is $\beta_{i j}=1 / 6$, the "transmission level" constraint is $\hat{P}^{o t g}=10^{-3}$, the single isolated cell capacity is $M A I_{0}=90$, and the exogenous rate for a border cell $z$ is $\lambda_{z}^{h o, e x}=0.25 l_{z}$, where $l_{z}$ are the number of border sides of cell $z$. In all results presented below, the constraint on the outage probability is respected.

The first configuration optimizes the allocation in a homogeneously loaded system with increasing load. Numerical results of the computation are plotted in both $\mathrm{A}$ and $\mathrm{B}$ cases, and are represented in Figure 3 (a), where blocking probabilities are plotted against the cell traffic load. The numerical scale of the hand-off blocking probability in case B is mapped on the right of the plot for a scaling problem. It is clear how, in case $\mathrm{B}$, the constraint over hand-off call blocking produces a slight increase in new call blocking probability.

Furthermore, the presence of a single hot-spot cell, having an increased new call generation rate, within a lightly loaded system, has been considered. The remaining cells present a homogeneous load of 20 Erlangs. Plots for an increasing traffic load of the hot-spot cell are presented, considering cases A and B. In Figure 3 (b) blocking probabilities are displayed as a function of the hot-spot cell load.

Another case of a single hot-spot cell has then been taken into account. Here the other cells in the system are homogeneously loaded at 50 Erlangs. Again, plots for an increasing traffic load of the hot-spot cell are presented, considering case A and B. In Figure 3 (c) blocking probabilities are displayed as a function of the hot-spot cell load.

Finally, the plot of blocking probabilities referring to the traffic lane is plotted in Fig 3 (d), again for both A and B cases, against an increasing load of the cells belonging to the traffic lane. The mobility coefficient relative to such cells has been increased to $\gamma_{i}=0.4$, and also the coefficients $\beta_{i j}$ of the sides marked by arrows in Fig 2 (b) are slightly increased.

\section{CONCLUSIONS}

A CDMA wireless cellular network has been considered, where the maximum capacity is fixed by a given level of multiple access interference. This global resource is dynamically assigned to different cells, according to the changing user mobility patterns and load variations. The assignment strategy is based on a two-level control, where a set of 

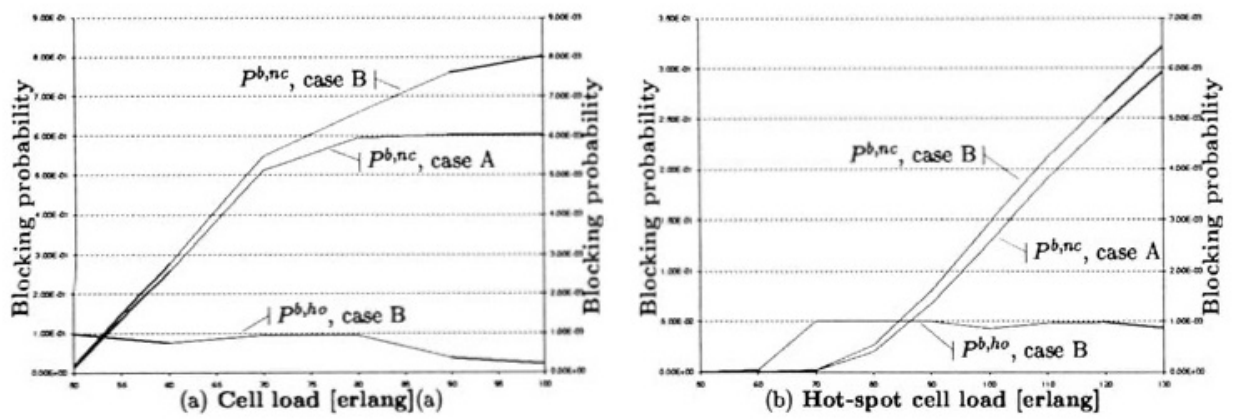

(b) Hot-spot cell load [erlang]
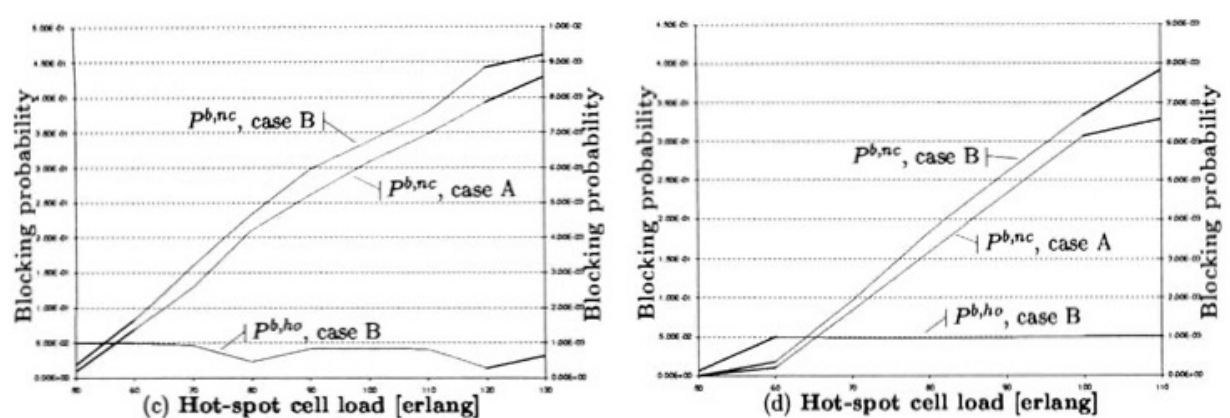

(d) Hot-spot cell load [erlang]

Figure 3 Results(read $P^{\text {b,ho }}$ values on the right scale) for: (a) homogeneous load (b) hot-spot "unloaded" (c) hot-spot "loaded" (d) traffic lane

thresholds implement the required resource distribution, whereas a controller within each cell decides on the admission of new calls. Numerical results show the effectiveness of the mechanism in keeping the handoff rejection level below a given upper bound, while always respecting the constraints on the outage probability.

\section{REFERENCES}

[1] Anastasi G., D. Grillo, L. Lenzini "An access protocol for speech/data/video integration in TDMA-based advanced mobile systems" IEEE J. Select. Areas Commun., vol. 15, no. 8, pp. 1498-1510, Oct. 1997.

[2] Bolla R., F. Davoli, C. Nobile "The RRA-ISA multiple access protocol with and without simple priority schemes for real-time and data traffic in wireless cellular systems" Mobile Networks and Applications, vol. 2, pp. 45-53, 1997.

[3] Bolla R., D. Budicin, F. Davoli, R. Rossi "A coordinated structure for call admission control and dynamic channel allocation in wireless cellular networks" Proc. IEEE Internat. Symp. on Wireless Commun. (ISWC'g9), Victoria, BC, Canada, June 1999 (Extended Abstract).

[4] Bolla R., F. Davoli "An iterative optimization scheme for resource adaptation to user mobility patterns in cellular wireless systems" to be presented at IEEE Wireless Communications and Networking Conference 2000 (WCNC'2000), Chicago, IL, Sept. 2000. 
[5] Liu T.K., J.A. Silvester "Joint admission/congestion control for wireless CDMA systems supporting integrated services" IEEE J. Select. Areas Commun., vol. 16, no. 6, pp. 845-857, Aug. 1998.

[6] Liu Z., M. El Zarki "SIR-Based Call Admission Control for DS-CDMA Cellular Systems" IEEE J. Select. Areas Commun., vol. 12, no. 4, pp.638-644, May 1994.

[7] Gilhousen K.S., I.M. Jacobs, R. Padovani,A.J. Viterbi, L.A. Weaver, Jr., C.E. Wheatley III "On the Capacity of a Cellular CDMA System" IEEE Trans. Vehic. Technol., vol. 40, no. 2, pp.303-311, May 1991.

[8] Mainkar V., K.S. Trivedi "Sufficient conditions for existence of a fixed point stochastic reward net-based iterative models" IEEE Trans. Softw. Engin., vol. 22, no. 9, pp. 640-653, Sept. 1996.

[9] Naghshineh M., A. S. Acampora "QoS provisionig in micro-cellular networks supporting multiple classes of traffic" Wireless Networks, vol. 2, pp. 195-203, 1996.

[10] Sampath A., J.M. Holtzman "Access control of data in integrated voice/data CDMA systems: benefits and tradeoffs" IEEE J. Select. Areas Commun., vol. 15 , no. 8, pp. 1511-1526, Oct. 1997.

[11] Samukic A. "UMTS Universal Mobile Telecommunications System: Development of standards for the third generation" IEEE Trans. Vehic. Technol., vol. 47, no. 4, pp. 1099-1104, Nov. 1998.

[12] Yu O.T.V., V.C.M. Leung "Adaptive resource allocation for prioritized call admission over an ATM-based wireless PCM" IEEE J. Select. Areas Commun., vol. 15 , no. 7 , pp. 1208-1225, Sept. 1997. 